\title{
Avaliação da correlação entre as concentrações de poluentes atmosféricos e a mortalidade de idosos no município de Curitiba
}

\section{Evaluation of the correlation between atmospheric pollutant concentrations and elderly mortality in Curitiba}

\author{
Guilherme Augusto Robles Esquivel \\ Engenheiro Químico pela Universidade Estadual de Maringá (UEM). Especialista em Gestão Ambiental pela UEM. Mestre em Gestão Ambiental pela \\ Universidade Positivo
}

\section{Júlio Gomes}

Engenheiro Civil pela Universidade Federal do Paraná (UFPR). Mestre em Engenharia Hidráulica pela UFPR. Doutor em Recursos Hídricos e Saneamento Ambiental pelo Instituto de Pesquisas Hidráulicas da Universidade Federal do Rio Grande do Sul (IPH/UFRGS). Professor do Programa de Pós-Graduação em Gestão Ambiental da Universidade Positivo

\section{Andreas Friedrich Grauer}

Engenheiro de Processo pela Universidade de Stuttgart. Doutor em Emissões Veiculares pela Universidade de Stuttgart. Professor do Mestrado Internacional em Meio Ambiente Urbano e Industrial da UFPR/Universidade de Stuttgart/SENAI-PR

\section{Resumo}

Este estudo tem por objetivo verificar as associações de curto prazo entre as concentrações de poluentes atmosféricos e a mortalidade de idosos no município de Curitiba (PR). O período de análise compreendeu os anos de 2003 a 2008. Foram realizadas análises de correlação simples e de regressão múltipla, utilizando-se um modelo aditivo generalizado (MAG), para avaliar as relações entre os poluentes dióxido de enxofre $\left(\mathrm{SO}_{2}\right)$, ozônio $\left(\mathrm{O}_{3}\right)$ e partículas totais em suspensão (PTS) e o número de óbitos totais (ObT) e por doenças respiratórias (ObR) de pessoas com idade superior a 60 anos. Os resultados obtidos mostraram relações positivas e estatisticamente significativas entre os níveis de PTS e a mortalidade de idosos por todas as causas (ObT), mesmo considerando-se que os níveis de PTS atenderam aos padrões de qualidade do ar para o período em estudo.

Palavras-chave: qualidade do ar; poluentes atmosféricos; mortalidade em idosos.

\section{Abstract}

This study aims to analyze the short-term association between concentration of atmospheric pollutants and the mortality of elderly in the city of Curitiba (Brazil). The data comprised the period from 2003 to 2008 . The relationships between the pollutants sulfur dioxide $\left(\mathrm{SO}_{2}\right)$, ozone $\left(\mathrm{O}_{3}\right)$ and total suspended particles ( $\left.\mathrm{TSP}\right)$ and the number of total deaths (ObT) and deaths due to respiratory deceases (ObR) of people aged over 60 years were investigated with simple correlation and multiple regression analyses, using a generalized additive model (GAM). The results showed a positive and statistically significant relationship between TSP concentrations and elderly mortality from all causes (ObT), even considering that TSP concentrations met the air quality standards during the period of study.

Keywords: air quality; atmospheric pollutants; elderly mortality.

\section{Introdução}

A poluição do ar tem sido associada ao aumento da procura por serviços de saúde, aumento no número de problemas de saúde e elevação no número de óbitos por diversas causas. Em grandes centros urbanos, a poluição do ar aliada a outros fatores, como saneamento e desigualdades sociais, contribui para a deterioração da qualidade de vida em grandes cidades de países em desenvolvimento (DANNIOLIVEIRA, 2008; BAKONYI, 2009).

Sicard et al. (2011) citam uma série de estudos que têm mostrado associações entre a exposição a poluentes clássicos (material particulado, ozônio, dióxido de enxofre e pólens) e a saúde humana de modo geral. Katsouyanni et al. (1995) destacam que os padrões de qualidade do ar são estabelecidos com base em episódios severos de 
poluição do ar, contudo estudos demonstraram que níveis baixos e moderados de poluição têm efeitos de curto prazo sobre a mortalidade e a morbidade e que esses efeitos são mensuráveis em níveis de poluição que não excedem os padrões estabelecidos.

Schwartz e Marcus (1990), Ackermann-Liebrich, Leuenberger Schuwartz et al. (1997), Clancy, Goodman, Sinclair et al. (2002), Junger, Leon e Mendonça (2005) e Ostro, Broadwin, Green et al. (2006) são exemplos de estudos que mostram correlação positiva entre a concentração de poluentes na atmosfera e efeitos sobre a saúde humana em diversas cidades do mundo, com efeitos observados para concentrações inferiores aos padrões de qualidade do ar vigentes. No Brasil também existem estudos similares que correlacionam indicadores de saúde à concentração de poluentes atmosféricos, como, por exemplo, Saldiva, Pope, Schwartz et al. (1995), Braun (2003), Daumas, Mendonça e Léon (2004), Bakonyi, Danni-Oliveira, Martins et al. (2004) e Arruda (2008).

Em geral, os efeitos à saúde humana são o somatório de exposições a diversos agentes, nem sempre fáceis de serem quantificados em estudos epidemiológicos. Embora os efeitos não estejam bem definidos nos indivíduos expostos, alguns efeitos agudos têm sido estudados em indivíduos previamente saudáveis e também em indivíduos portadores de doença respiratória ou cardiovascular crônica. Os grupos mais afetados, em geral, são crianças e idosos (KINNEY e OZKAYNAK, 1991; SALDIVA et al., 1995; FREITAS et al., 2004; FILLEUL et al., 2004; CANÇADO et al., 2006; OSTRO et al., 2006; DANNI-OLIVEIRA, 2008; TRESMONDI et al., 2008).

A verificação da associação entre a mortalidade de idosos e as concentrações de certos poluentes pode ser uma ferramenta útil na definição de ações preventivas e políticas públicas, visando o controle e monitoramento das emissões atmosféricas. Os trabalhos que associam a poluição do ar a indicadores de saúde também possibilitam a realização de previsões para demanda de serviços de saúde, em caso de situações de deterioração da qualidade do ar.

Dentro deste contexto, o objetivo do presente trabalho foi verificar a associação de curto prazo entre as concentrações dos principais poluentes atmosféricos e a mortalidade de idosos no município de Curitiba. O referido município é carente de estudos desta natureza, principalmente considerando-se que os idosos constituem um dos grupos mais suscetíveis aos efeitos da poluição do ar sobre a saúde humana.

\section{Métodos}

No presente estudo, analisou-se a relação entre as concentrações de poluentes atmosféricos e os óbitos ocorridos por todas as causas (ObT) e os óbitos ocorridos por causas respiratórias (ObR), por constituírem os efeitos mais frequentemente associados à poluição do ar. O período de análise compreendeu os anos de 2003 a 2008. A área de estudo correspondeu ao município de Curitiba (PR).
Os dados de óbitos de idosos foram fornecidos pela Secretaria Municipal da Saúde de Curitiba, constando data do óbito e causa da morte, segundo a Classificação Internacional de Doenças - CID (OMS, 2000). Considerou-se como idoso, a pessoa com idade igual ou superior a 60 anos, conforme a definição apresentada no Estatuto do Idoso (BRASIL, 2003).

Em relação à qualidade do ar, foram usadas as concentrações de poluentes observadas nas estações amostradoras localizadas na Praça Ouvidor Pardinho e na Santa Casa, ambas na região central de Curitiba e distantes 920 m, fornecidas pelo Instituto Ambiental do Paraná (IAP). A estação Santa Casa apresenta leituras diárias de fumaça, dióxido de enxofre $\left(\mathrm{SO}_{2}\right)$ e partículas totais em suspensão (PTS), obtidas por amostrador manual. Já a estação Praça Ouvidor Pardinho é uma estação automática, onde são amostrados diariamente $\mathrm{SO}_{2}$, PTS, dióxido de nitrogênio $\left(\mathrm{NO}_{2}\right)$, ozônio $\left(\mathrm{O}_{3}\right)$, monóxido de carbono (CO) e partículas inaláveis (PI). Foram também levantados os dados de temperatura média diária (Temp) e umidade média diária (Umid) observados na estação Praça Ouvidor Pardinho para representação das variáveis meteorológicas. As concentrações de alguns poluentes foram fornecidas em ppb e foram transformadas para $\mu \mathrm{g} \cdot \mathrm{m}^{-3}$, admitindo-se, por hipótese, uma condição de pressão de 1 atm e de temperatura de $25^{\circ} \mathrm{C}$, condição de referência sugerida em CONAMA (1990).

A primeira etapa do estudo consistiu em uma análise preliminar dos dados com o objetivo de caracterizar o comportamento das séries de poluentes e de óbitos em escala diária, mensal e anual. Inicialmente, as séries foram analisadas em relação ao número de dias com falhas de observação para definir as séries utilizadas no estudo. Optou-se por trabalhar com séries de poluentes com, no máximo, $30 \%$ de falhas de observação para o período de estudo.

Ainda em relação a esta primeira etapa, realizou-se uma análise conjunta do número de óbitos e das variáveis meteorológicas (Temp e Umid) com os objetivos de verificar o comportamento destas variáveis no período de estudo e de analisar a possível interferência das variáveis meteorológicas sobre o número de óbitos para a definição ou não das variáveis meteorológicas como variáveis de confusão dentro do modelo aditivo generalizado (MAG). A análise foi feita de modo visual, procurando-se observar a variação ao longo do ano da razão das médias mensais pela média anual do número de óbitos e da razão das médias mensais pela média anual das variáveis meteorológicas.

A próxima etapa do estudo consistiu na análise de correlação simples entre os dados brutos, considerando-se como variáveis independentes, as séries de concentrações médias diárias de PTS e de $\mathrm{SO}_{2}$ nas estações Santa Casa e Praça Ouvidor Pardinho (PTS_SC, PTS_ $\mathrm{OP}, \mathrm{SO}_{2} \mathrm{SC}$ e $\mathrm{SO}_{2}$ OP), de $\mathrm{O}_{3}$ na estação Praça Ouvidor Pardinho $\left(\mathrm{O}_{3}\right.$ OP) e de Temp e Umid na estação Praça Ouvidor Pardinho. Como variáveis dependentes, adotou-se as séries de números diários de ObT e de ObR. O grau de correlação entre as variáveis foi estimado pelo cálculo do coeficiente de correlação de Pearson. 
$\mathrm{Na}$ etapa seguinte, procurou-se analisar a correlação simples entre os dados tratados. O tratamento dos dados consistiu no alisamento (ou suavização) das séries de óbitos e das variáveis meteorológicas temperatura e umidade. As séries de óbitos foram suavizadas com a aplicação da função smoothing do software estatístico SPSS - versão 16.0 (FIELD, 2009). Para as séries de dados de temperatura e umidade foi utilizado como alisador a média móvel de 30 dias. Os dados de concentração de poluentes não sofreram processo de alisamento, procurando-se, deste modo, manter o padrão de exposição mais próximo do realmente observado.

A última etapa consistiu da análise de regressão múltipla, onde se adotou um MAG. Estes modelos são uma boa opção para representar a sazonalidade e a interferência das variáveis meteorológicas. Neste tipo de modelo são usadas funções que possuem o mesmo domínio da função original, estimadas através de curvas de alisamento. A curva alisada nada mais é do que uma média de certo número de valores na vizinhança de determinado valor (CONCEIÇÃO; SALDIVA; SINGER, 2001; TADANO; UGAYA; FRANCO, 2009).

Segundo Conceição; Saldiva; Singer (2001) e Tadano; Ugaya; Franco (2009), em estudos de impacto da poluição atmosférica na saúde humana nem sempre é possível a utilização de um modelo de regressão simples devido ao caráter não linear das variáveis de resposta. Neste caso, utilizam-se modelos que fornecem uma alternativa para a transformação de dados, como os MAG (HASTIE e TIBSHIRANI, 1990)

$\mathrm{Na}$ etapa de análise de regressão múltipla, os diferentes modelos foram sempre constituídos com três variáveis independentes, sendo duas delas, as variáveis meteorológicas (temperatura e umidade), consideradas como variáveis de confusão, e a terceira sendo a concentração do poluente, cuja relação com a variável dependente (número de óbitos) se buscava analisar. Optou-se por analisar todas as relações possíveis entre número de óbitos e concentração de poluentes, independentemente dos resultados da análise de correlação linear simples, em razão da possível influência das variáveis de confusão na relação entre as variáveis.

Em função dos resultados obtidos na etapa de análise de regressão múltipla, estimou-se o risco relativo (RR) da mortalidade de idosos (número de óbitos totais) associadas à diminuição ou ao aumento da concentração de PTS na estação Santa Casa que apresentou maiores concentrações em comparação à estação Praça Ouvidor Pardinho.
Para a estimativa do RR, calculou-se inicialmente a variação relativa do número de ObT em função da variação absoluta da concentração média de PTS na estação Santa Casa, utilizando-se a equação a seguir, adaptada de Freitas et al. (2004):

$\Delta$ obitos $=100\left(e^{\beta . \Delta c}-1\right)$

Equação 1

Onde:

$\Delta$ obitos: variação relativa no número de óbitos (\%);

$\beta$ : coeficiente de regressão obtido no modelo de regressão múltipla utilizado;

$\Delta c$ : variação absoluta na concentração média do poluente $\left(\mu \mathrm{g} \cdot \mathrm{m}^{-3}\right)$.

Os valores de variação absoluta na concentração média do poluente $(\Delta \mathrm{c})$ foram calculados adotando-se variações relativas de $\pm 5 \mathrm{e}$ $\pm 10 \%$ sobre a média para o período das concentrações médias diárias de PTS na estação Santa Casa. Um cenário mais pessimista também foi adotado, assumindo-se um aumento da concentração média do percentil 10 para o percentil 90, considerando-se a distribuição de frequências das concentrações médias diárias de PTS na estação Santa Casa, obtida para o período de 2003 a 2008

O RR foi então estimado, sendo entendido como a relação entre o número de óbitos totais em uma condição futura de poluição e o número de óbitos totais na condição atual. Matematicamente, de modo simples, o RR foi estimado por:

$\mathrm{RR}=\left(1+\frac{\Delta \text { obitos }}{100}\right)$

Equação 2

Onde:

$\triangle$ obitos: variação relativa no número de óbitos (\%);

RR: risco relativo.

\section{Resultados}

A análise preliminar dos dados mostrou que apenas as séries de PTS e $\mathrm{SO}_{2}$ nas estações Santa Casa e Praça Ouvidor Pardinho e de $\mathrm{O}_{3}$ na estação Praça Ouvidor Pardinho apresentaram índices de falhas inferiores a 30\% para o período de 2003 a 2008. Portanto, as séries de fumaça na estação Santa Casa e de $\mathrm{NO}_{2}$, $\mathrm{CO}$ e PI na estação Praça Ouvidor Pardinho não foram analisadas no estudo. A Tabela 1 apre-

Tabela 1 - Níveis dos poluentes $\left(\mu \mathrm{g} \cdot \mathrm{m}^{-3}\right)$ observados nas estações Santa Casa (SC) e Praça Ouvidor Pardinho (OP) durante 0 período de 2003 a 2008

\begin{tabular}{|c|c|c|c|c|c|c|c|c|c|c|}
\hline \multirow{2}{*}{ poluente/estação } & \multirow{2}{*}{$n$} & \multirow{2}{*}{ média $^{1}$} & \multirow{2}{*}{ desvio padrão } & \multirow{2}{*}{ mínimo } & \multicolumn{5}{|c|}{ Percentis } & \multirow{2}{*}{ máximc } \\
\hline & & & & & 10 & 25 & 50 & 75 & 90 & \\
\hline PTS-SC & 2.010 & 76,1 & 29,1 & 5,0 & 45,0 & 56,0 & 72,0 & 91,0 & 114,0 & 231,0 \\
\hline PTS-OP & 1.801 & 28,9 & 23,5 & 0,9 & 9,7 & 13,8 & 21,4 & 35,0 & 59,5 & 168,3 \\
\hline $\mathrm{SO}_{2}-\mathrm{SC}$ & 2.146 & 22,1 & 12,0 & 0,0 & 6,0 & 13,0 & 22,0 & 30,0 & 37,0 & 80,0 \\
\hline $\mathrm{SO}_{2}-\mathrm{OP}$ & 2.149 & 4,8 & 9,2 & 0,0 & 0,2 & 0,9 & 2,6 & 6,0 & 10,3 & 256,4 \\
\hline $\mathrm{O}_{3}-\mathrm{OP}$ & 2.112 & 24,9 & 10,5 & 0,3 & 12,4 & 17,6 & 23,8 & 31,8 & 39,3 & 73,1 \\
\hline
\end{tabular}

PTS: partículas totais em suspensão; $\mathrm{SO}_{2}$ : dióxido de enxofre; $\mathrm{O}_{3}$ : ozônio; ${ }^{1}$ média aritmética. 
senta um resumo das estatísticas relacionadas às séries de poluentes utilizadas no estudo.

As concentrações médias anuais (médias geométricas) de PTS observadas nas estações Santa Casa e Praça Ouvidor Pardinho para o período de 2003 a 2008 foram da ordem de $70 \mu \mathrm{g} \cdot \mathrm{m}^{-3}$ e $25 \mu \mathrm{g} \cdot \mathrm{m}^{-3}$, respectivamente. A Figura 1 apresenta as concentrações médias mensais (médias geométricas) de PTS observadas nas estações Santa Casa e Praça Ouvidor Pardinho para o período de estudo.

As concentrações médias anuais de $\mathrm{SO}_{2}$ na estação Santa Casa apresentaram uma redução de aproximadamente $30 \mu \mathrm{g} \cdot \mathrm{m}^{-3}$ para 15 Hg. $\mathrm{m}^{-3}$ entre 2003 e 2008. Na estação Praça Ouvidor Pardinho, as concentrações médias anuais de $\mathrm{SO}_{2}$ se mostraram sempre inferiores a $15 \mu \mathrm{g} \cdot \mathrm{m}^{-3}$ durante todo o período do estudo. A Figura 2 apresenta as concentrações médias mensais de $\mathrm{SO}_{2}$ observadas nas estações Santa Casa e Praça Ouvidor Pardinho para o período em estudo.

Em relação ao $\mathrm{O}_{3}$, as concentrações médias anuais observadas na estação Praça Ouvidor Pardinho variaram entre $20 \mu \mathrm{g} \cdot \mathrm{m}^{-3}$ e $30 \mu \mathrm{g} \cdot \mathrm{m}^{-3}$ para o período de 2003 a 2008, enquanto as maiores concentrações médias mensais foram da ordem de $40 \mu \mathrm{g} \cdot \mathrm{m}^{-3}$. A Figura 3 apresenta as concentrações médias mensais de $\mathrm{O}_{3}$ observadas na estação Praça Ouvidor Pardinho para o período em estudo.

A Figura 4 mostra as razões entre as médias mensais e a média anual do número de ObT e do número de ObR e as razões entre as médias mensais e as médias anuais de temperatura e de umidade.

A Tabela 2 apresenta os resultados das análises de correlação simples entre os dados brutos, utilizando-se o software SPSS versão 16.0, onde se destacam, em negrito, os coeficientes de correlação de Pearson do número diário de ObT e do número diário de ObR com as demais variáveis.

A Tabela 3 apresenta os resultados das análises de correlação simples entre os dados tratados, também usando-se o software SPSS versão 16.0, onde uma vez mais se destacam em negrito os coeficientes de correlação de Pearson do número diário de óbitos por todas as causas (ObT_s) e do número diário de óbitos por doenças respiratórias (ObR_s) em relação às demais variáveis

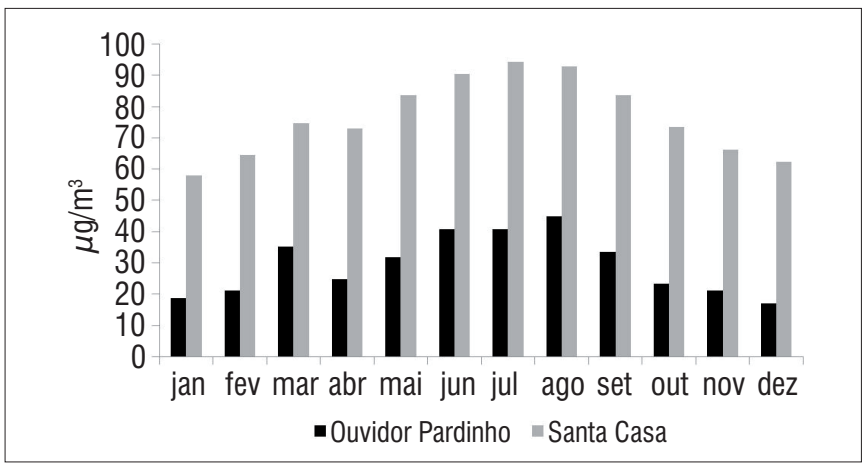

Figura 1 - Concentrações médias mensais de partículas totais em suspensão observadas nas estações Santa Casa e Praça Ouvidor Pardinho para o período 2003-2008.

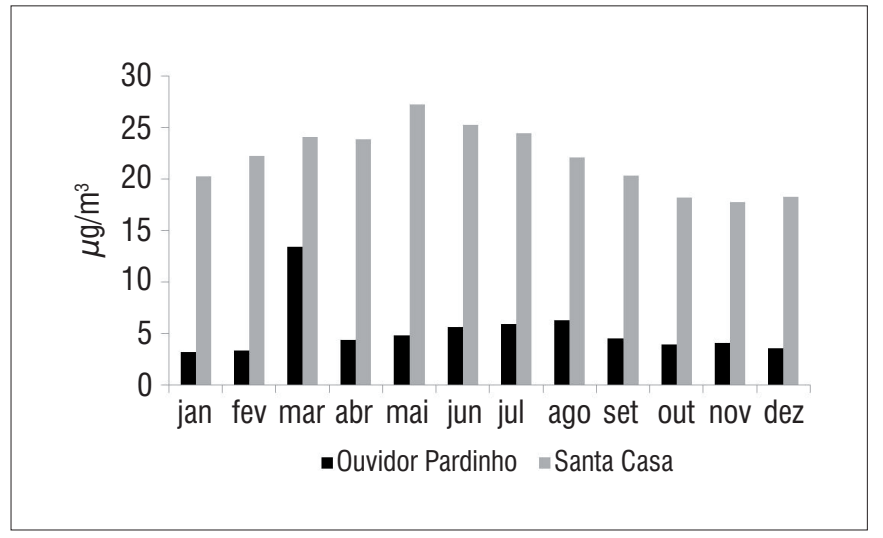

Figura 2 - Concentrações médias mensais de dióxido de enxofre observadas nas estações Santa Casa e Praça Ouvidor Pardinho para 0 período 2003-2008.

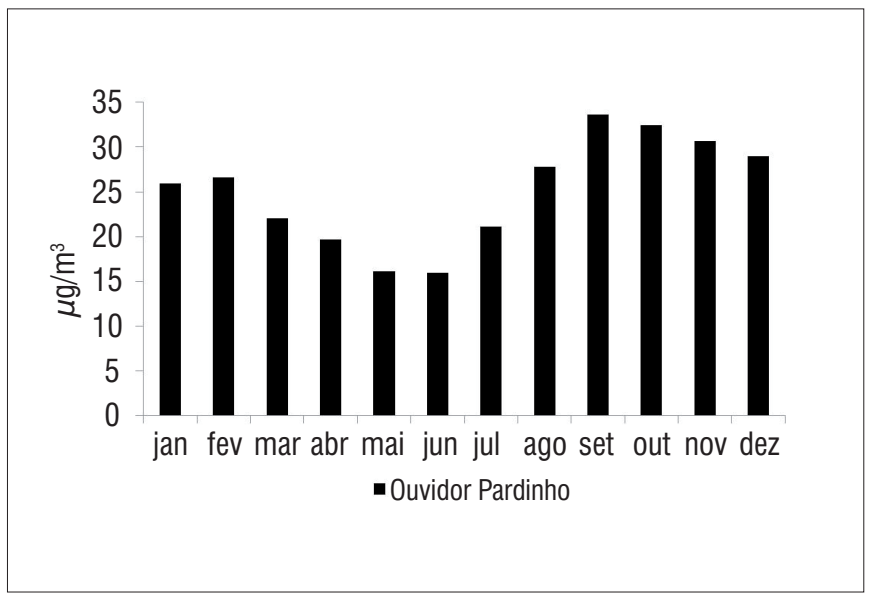

Figura 3 - Concentrações médias mensais de ozônio observadas na estação Praça Ouvidor Pardinho para o período 2003-2008.

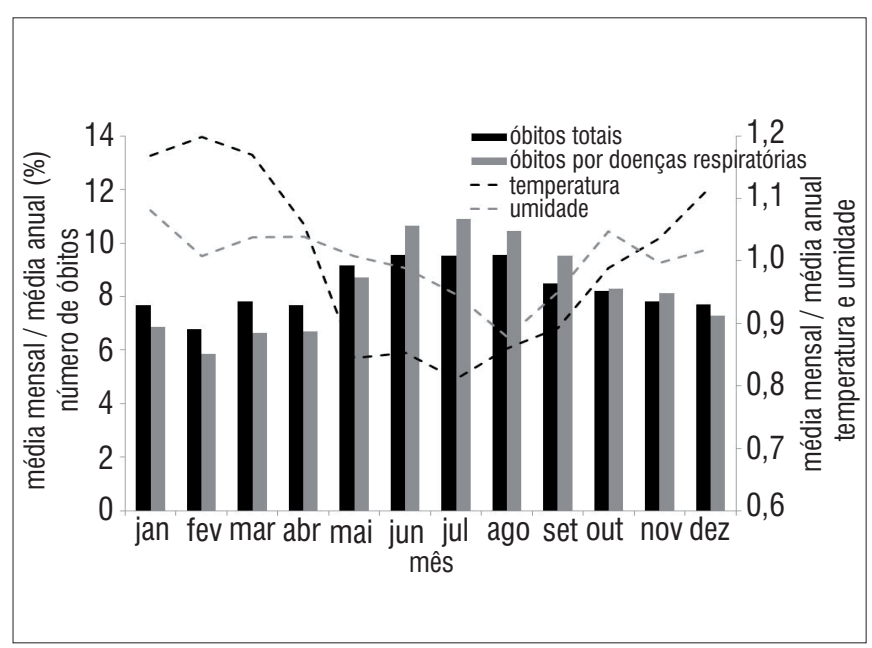

Figura 4 - Razões entre os números médios mensais e o número médio anual de óbitos, e razões entre as médias mensais e as médias anuais de temperatura e umidade. 
Tabela 2 - Coeficiente de correlação de Pearson entre os dados brutos

\begin{tabular}{|c|c|c|c|c|c|c|c|}
\hline Variável & PTS_OP & PTS_SC & $\mathrm{SO}_{2} \mathrm{OP}$ & $\mathrm{SO}_{2} \mathrm{SC}$ & $\mathrm{O}_{3} \mathrm{OP}$ & Temp & Umid \\
\hline $\mathrm{ObT}$ & $0,151^{* *}$ & $0,196^{\star *}$ & 0,039 & 0,006 & $-0,060 * *$ & $-0,198^{* *}$ & $-0,140 * *$ \\
\hline ObR & $0,107^{* *}$ & $0,145^{\star *}$ & 0,010 & $-0,003$ & $-0,016$ & $-0,151^{\star *}$ & $-0,112^{\star *}$ \\
\hline PTS_OP & 1 & $0,608^{\star *}$ & $0,181^{* *}$ & $0,140^{* *}$ & 0,003 & $0,104^{\star *}$ & $-0,512^{* *}$ \\
\hline PTS_SC & $0,608^{\star *}$ & 1 & $0,145^{\star *}$ & $0,121^{* *}$ & $0,050^{*}$ & $-0,004$ & $-0,451^{* *}$ \\
\hline $\mathrm{SO}_{2} \mathrm{OP}$ & $0,181^{\star *}$ & $0,145^{\star *}$ & 1 & 0,010 & $-0,086^{\star \star}$ & 0,003 & $-0,192^{* *}$ \\
\hline $\mathrm{SO}_{2-} \mathrm{SC}$ & $0,140^{\star *}$ & $0,121^{* *}$ & 0,010 & 1 & $-0,100$ ** & $-0,010$ & $-0,021$ \\
\hline $0_{3} \mathrm{OP}$ & 0,003 & $0,050^{*}$ & $-0,086^{\star *}$ & $-0,100^{* *}$ & 1 & $0,330 * *$ & $-0,338^{* *}$ \\
\hline Temp & $0,104^{\star *}$ & $-0,004$ & 0,003 & $-0,010$ & 0,330 ** & 1 & $-0,105^{\star *}$ \\
\hline
\end{tabular}

PTS: partículas totais em suspensão; $\mathrm{SO}_{2}$ : dióxido de enxofre; $\mathrm{O}_{3}$ : ozônio; SC: Santa Casa; OP: Praça Ouvidor Pardinho; Temp: temperatura média diária; Umid: umidade média diária; ObT: óbitos totais; ObR: óbitos por doenças respiratórias; *correlação significativa para $\alpha=5 \%$; ${ }^{\star *}$ correlação significativa para $\alpha=1 \%$.

Tabela 3 - Coeficiente de correlação de Pearson entre os dados tratados

\begin{tabular}{|c|c|c|c|c|c|c|c|}
\hline Variável & PTS_OP & PTS_SC & $\mathrm{SO}_{2} \mathrm{OP}$ & $\mathrm{SO}_{2} \mathrm{SC}$ & $0_{3} \mathrm{OP}$ & MV_Temp & MV_Umic \\
\hline ObT_s & $0,234^{\star *}$ & 0,290 ** & $0,058^{* *}$ & 0,005 & $-0,118^{\star *}$ & $-0,608^{* *}$ & $-0,208^{\star *}$ \\
\hline ObR_s & $0,194^{\star *}$ & $0,220^{\star *}$ & 0,041 & $-0,029$ & $-0,043^{*}$ & $-0,517^{\star \star}$ & $-0,221^{\text {** }}$ \\
\hline PTS_OP & 1 & $0,608^{\star *}$ & $0,181^{\text {** }}$ & $0,140^{* *}$ & 0,003 & $-0,304^{\star *}$ & $-0,286^{\star *}$ \\
\hline PTS_SC & $0,608^{\star *}$ & 1 & $0,145^{\star \star}$ & 0,121 ** & $0,050^{*}$ & $-0,386^{\star *}$ & $-0,178^{* *}$ \\
\hline $\mathrm{SO}_{2-} \mathrm{OP}$ & $0,181^{* *}$ & $0,145^{\star *}$ & 1 & 0,010 & $-0,086^{* *}$ & $-0,039$ & $-0,090$ ** \\
\hline $\mathrm{SO}_{2-} \mathrm{SC}$ & $0,140 * *$ & $0,121^{\star *}$ & 0,010 & 1 & $-0,100^{\star * *}$ & 0,031 & $0,095^{\star *}$ \\
\hline $\mathrm{O}_{3} \mathrm{OP}$ & 0,003 & $0,050^{*}$ & $-0,086^{\star *}$ & $-0,100^{\star *}$ & 1 & $0,067^{\star *}$ & $-0,164^{\star *}$ \\
\hline MV_Temp & $-0,304^{\star *}$ & $-0,386 * \star$ & $-0,039$ & 0,031 & $0,067^{\star *}$ & 1 & $0,268^{\star \star}$ \\
\hline
\end{tabular}

PTS: partículas totais em suspensão; $\mathrm{SO}_{2}$ : dióxido de enxofre; $\mathrm{O}_{3}$ : ozônio; SC: Santa Casa; OP: Praça Ouvidor Pardinho; MV Temp: média móvel da temperatura média diária; MV Umid: média móvel da umidade média diária; ObT_s: série alisada do número de óbitos totais; ObR s: série alisada do número de óbitos por doenças respiratórias; * ${ }^{\circ}$ Correlação significativa para $\alpha=5 \%$; ** correlação significativa para $\alpha=1 \%$.

Tabela 4 - Coeficiente de regressão obtido na análise de regressão múltipla

$\begin{array}{lccccc}\text { Variável } & \text { PTS_OP } & \text { PTS_SC } & \mathrm{SO}_{2 \_} \mathrm{OP} & \mathrm{SO}_{2-} \mathrm{SC} & \mathrm{O}_{3-} \mathrm{OP} \\ \text { ObT_s } & 0,007 & 0,007 & 0,008^{*} & 0,010 & -0,023 \\ \text { ObR_s } & 0,002^{*} & 0,001^{*} & 0,004^{*} & 0,000^{*} & 0,000^{*}\end{array}$

PTS: partículas totais em suspensão; $\mathrm{SO}_{2}$ : dióxido de enxofre; $\mathrm{O}_{3}$ : ozônio; SC: Santa Casa; OP: Praça Ouvidor Pardinho; ObT_s: série alisada do número de óbitos totais; ObR_s: série alisada do número de óbitos por doenças respiratórias; *correlação não significativa para $\alpha=5 \%$.

A Tabela 4 apresenta os coeficientes de regressão obtidos para os diferentes modelos, considerando-se o uso do MAG. Não são apresentados os coeficientes de regressão relativos às variáveis meteorológicas por serem consideradas como variáveis de confusão no MAG.

A Tabela 5 apresenta as estimativas do RR para ObT, utilizandose as Equações 1 e 2 e adotando-se, como condição atual, o valor de $76,1 \mu \mathrm{g} \cdot \mathrm{m}^{-3}$ para a concentração média de PTS na estação Santa Casa (PTS_SC) e o coeficiente de regressão entre ObT e PTS_SC apresentado na Tabela 4.

\section{Discussão}

Os valores de PTS e $\mathrm{SO}_{2}$ observados nas estações Santa Casa e Praça Ouvidor Pardinho apresentaram uma diferença significativa, como observado nas Figuras 1 e 2. Em todos os meses, verificaramse concentrações médias de PTS e $\mathrm{SO}_{2}$ na estação Santa Casa significativamente superiores aos valores observados na estação Praça
Ouvidor Pardinho. Um fator que provavelmente colabora para esta diferença é o intenso fluxo de veículos do ciclo diesel, utilizados no transporte coletivo do município de Curitiba, que trafegam na Praça Rui Barbosa, em frente à Santa Casa

Verificou-se também uma aparente sazonalidade nas concentrações de PTS em ambas as estações, com tendência a maiores valores de concentração, no inverno, e menores valores de concentração, no verão. No caso do $\mathrm{SO}_{2}$, observou-se uma aparente sazonalidade nos valores amostrados na estação Santa Casa, apresentando o mesmo padrão de PTS. Não se verificou sazonalidade para os valores de $\mathrm{SO}_{2}$ observados na estação Praça Ouvidor Pardinho.

O padrão primário para o PTS para média geométrica anual $\left(80 \mu \mathrm{g} . \mathrm{m}^{-3}\right)$ não foi excedido durante o período em estudo. Contudo, as concentrações médias anuais na estação Santa Casa resultaram em um patamar próximo de $80 \mu \mathrm{g} \cdot \mathrm{m}^{-3}$. O padrão primário para PTS para média de $24 \mathrm{~h}\left(240 \mu \mathrm{g} \cdot \mathrm{m}^{-3}\right)$ não foi excedido durante todo o período em estudo. O padrão primário para $\mathrm{O}^{\mathrm{SO}_{2}}$ para média anual $\left(80 \mu \mathrm{g} \cdot \mathrm{m}^{-3}\right)$ foi excedido no ano de 2003, na estação Santa Casa, que apresentou média anual de $82 \mu \mathrm{g} \cdot \mathrm{m}^{-3}$. O padrão primário para as concentrações médias de $24 \mathrm{~h}$ de $\mathrm{SO}_{2}\left(365 \mu \mathrm{g} \cdot \mathrm{m}^{-3}\right)$ não foi excedido nenhuma vez durante todo o período em estudo.

Em relação ao $\mathrm{O}_{3}$, amostrado somente na Praça Ouvidor Pardinho, as maiores concentrações médias mensais foram da ordem de $40 \mu \mathrm{g} \cdot \mathrm{m}^{-3}$. Como os dados obtidos referem-se a concentrações diárias, não foi possível verificar se houve violação ao padrão, já que o mesmo é definido para média horária. As concentrações de $\mathrm{O}_{3}$ 
Tabela 5 - Estimativas da variação do número de óbitos totais e do risco relativo em função da variação na concentração de partículas totais em suspensão na estação Santa Casa

\begin{tabular}{|c|c|c|c|}
\hline $\begin{array}{l}\text { Variação relativa na concentração } \\
\text { média do poluente (\%) }\end{array}$ & $\begin{array}{l}\text { Variação absoluta na concentração } \\
\text { média do poluente }\left(\mu \mathrm{g} \cdot \mathrm{m}^{-3}\right)\end{array}$ & $\begin{array}{l}\text { Variação relativa no número } \\
\text { de óbitos totais (\%) }\end{array}$ & Risco relativo \\
\hline-10 & $-7,6$ & $-5,2$ & 0,948 \\
\hline-5 & $-3,8$ & $-2,6$ & 0,974 \\
\hline+5 & 3,8 & 2,7 & 1,027 \\
\hline+10 & 7,6 & 5,5 & 1,055 \\
\hline $10-90^{1}$ & 69,0 & 62,1 & 1,621 \\
\hline
\end{tabular}

Risco relativo associado ao aumento da concentração média do percentil 10 para o percentil 90.

observadas na estação Praça Ouvidor Pardinho mostraram uma aparente sazonalidade. Contudo, neste caso, com tendência de maiores valores no verão e menores valores, no inverno, o que pode ser explicado pelo fato do $\mathrm{O}_{3}$ ser um poluente secundário, formado através de reações fotoquímicas na atmosfera, sendo sua formação influenciada pela radiação solar, mais intensa no verão.

A análise visual da relação entre as razões das médias mensais e da média anual dos números de óbitos e as razões das médias mensais e das médias anuais das variáveis meteorológicas (Figura 4), mostrou uma aparente sazonalidade das variáveis ObT e ObR e uma correlação negativa das referidas variáveis com as variáveis meteorológicas (Temp e Umid). Este comportamento justificou a inclusão da temperatura e da umidade como variáveis de confusão no MAG.

Os resultados das análises de correlação simples entre os dados brutos mostraram correlações positivas e significativas entre os números de ObT e ObR e as concentrações de PTS (PTS_OP e PTS_SC). Não se verificaram correlações significativas entre os números de ObT e ObR e as concentrações de $\mathrm{SO}_{2}\left(\mathrm{SO}_{2}-\mathrm{OP}\right.$ e $\left.\mathrm{SO}_{2} \mathrm{SC}\right)$. Destaca-se a existência de correlação negativa e significativa entre o número de ObT e a concentração de $\mathrm{O}_{3}\left(\mathrm{O}_{3}-\mathrm{OP}\right)$, o que parece incoerente dada a sua condição de poluente atmosférico.

Verificou-se, comparando-se os resultados das Tabelas 2 e 3, que o tratamento dos dados implicou no aparecimento de correlação positiva e significativa entre o número de $\mathrm{ObT}$ e a concentração de $\mathrm{SO}_{2}$ na estação Praça Ouvidor Pardinho $\left(\mathrm{SO}_{2} \mathrm{OP}\right)$ e de correlação negativa e significativa entre o número de ObR e a concentração de $\mathrm{O}_{3}$ na estação Praça Ouvidor Pardinho ( $\mathrm{O}_{3}$ OP). Verificaram-se também, de modo geral, maiores valores em módulo de correlação entre ObT_s e ObR_s com as demais variáveis. Importante destacar o alto grau de correlação entre ObT_s e ObR_s com a média móvel da temperatura média diária (MV_Temp).

Ainda em relação às análises de correlação simples, é importante destacar alguns coeficientes de correlação observados entre as variáveis independentes. Observou-se uma correlação positiva, significativa e relativamente alta entre as concentrações de PTS nas duas estações (PTS_SC e PTS_OP). Este resultado é importante, uma vez que a análise preliminar havia mostrado valores de concentração de PTS na estação Santa Casa superiores aos observados na estação Praça Ouvidor Pardinho. O mesmo não ocorre quando se analisa a correlação entre os valores de concentração de $\mathrm{SO}_{2}\left(\mathrm{SO}_{2}-\mathrm{OP}\right.$ e $\left.\mathrm{SO}_{2} \mathrm{SC}\right)$, onde, além de serem observadas concentrações de $\mathrm{SO}_{2}$ superiores na estação Santa Casa, verificou-se também a ausência de correlação entre os valores observados nas referidas estações. A existência de correlação positiva entre as concentrações de $\mathrm{O}_{3}\left(\mathrm{O}_{3}-\mathrm{OP}\right)$ e a Temp ajudam a explicar o resultado da correlação negativa entre $\mathrm{ObT}$ e $\mathrm{O}_{3}$.

A análise de regressão múltipla, conforme os resultados apresentados na Tabela 4, mostrou coeficientes de regressão significativos somente para o número de ObT_s nos modelos tendo como variáveis independentes, além das variáveis meteorológicas (variáveis de confusão), PTS_OP, PTS_SC, SO $\mathrm{S}_{2}$ SC e O $\mathrm{O}_{3}$ OP. Destaca-se que, no modelo envolvendo $\mathrm{O}_{3}$-OP, como variável independente, o coeficiente de regressão obtido foi negativo, mesmo com a inclusão das variáveis meteorológicas, o que novamente parece ser incoerente dada a condição de poluente atmosférico do $\mathrm{O}_{3}$.

No Brasil, estudos anteriores obtiveram correlações positivas e significativas entre as concentrações de poluentes atmosféricos e a mortalidade (SALDIVA et al., 1995; CONCEIÇÃO et al., 2001; BRAUN, 2003; DAUMAS; MENDONÇA e LÉON, 2004; FREITAS et al., 2004). Os resultados também são corroborados por estudos em outros países, como por exemplo, Schwartz e Marcus (1990), Dockery et al. (1993), Ackermann-Liebrich et al. (1997), Clancy et al. (2002) e Ostro et al. (2006).

O RR para o número de ObT para incrementos de 5 e 10\% na concentração média de PTS na estação Santa Casa resultou, respectivamente, em 1,027 e 1,055, representando aumento de 2,7 e 5,5\% no número de ObT. O aumento da concentração de PTS na estação Santa Casa do percentil 10 para o percentil 90 resultou em um RR de 1,621, correspondendo a um aumento de 62,1\% no número de ObT. Este valor é superior ao encontrado em trabalhos semelhantes realizados em outras cidades do país, como por exemplo, Saldiva et al. (1995); Conceição et al. (2001), Freitas et al. (2004). Destaca-se que a variação da concentração média de PTS na estação Santa Casa do percentil 10 para o percentil 90 implicaria no aumento da referida concentração de $45 \mu \mathrm{g} \cdot \mathrm{m}^{-3}$ para $114 \mu \mathrm{g} \cdot \mathrm{m}^{-3}$, o que não representa um cenário realista no curto prazo.

Uma característica de estudos epidemiológicos, envolvendo a análise de séries temporais, como o presente trabalho, é que o seu 
desenvolvimento não implica em um aumento no conhecimento sobre o mecanismo responsável pela interação entre o nível de poluição e a saúde humana. A análise de correlações entre as variáveis não permite estabelecer uma relação de causa e efeito entre elas.

A definição de modelos de regressão, envolvendo apenas um dos poluentes atmosféricos no conjunto de variáveis independentes, não contempla a hipótese de uma possível sinergia entre os diferentes poluentes no tocante aos efeitos sobre a saúde humana.

Outra limitação deste tipo de estudo se refere à questão de qual é realmente o nível de poluição a que a população está exposta. A densidade das redes amostradoras de qualidade do ar na cidade de Curitiba, e na maioria das cidades do país, é relativamente pequena para permitir a caracterização da variabilidade espacial da concentração de poluentes e a estimativa de um valor mais real do nível de poluição ao qual a população está efetivamente exposta.

Um aspecto a se destacar é o de que a comparação das estatísticas das séries dos poluentes observados em ambas as estações (PTS e $\mathrm{SO}_{2}$ ) e a correlação entre os valores observados de um mesmo poluente em ambas as estações, notadamente o $\mathrm{SO}_{2}$, sugerem um estudo mais aprofundado sobre a confiabilidade dos dados, inclusive em razão dos diferentes métodos de obtenção das concentrações (estação manual versus estação automática).

\section{Conclusões}

O presente estudo procurou avaliar a existência de associação de curto prazo entre as concentrações dos principais poluentes presentes na atmosfera e a mortalidade de idosos no município de Curitiba. Foram analisadas séries de números de ObT e ObR e séries de concentrações diárias de PTS e $\mathrm{SO}_{2}$, relativas às estações amostradoras do ar Santa Casa e Praça Ouvidor Pardinho, localizadas na região central de Curitiba. Analisou-se também a série de concentrações diárias de $\mathrm{O}_{3}$, observadas na estação Praça Ouvidor Pardinho. O período dos dados compreendeu os anos de 2003 a 2008.

Os resultados obtidos mostraram a existência de coeficientes de regressão positivos e significativos entre o número de ObT e a concentração diária de PTS nas duas estações analisadas e entre o número de ObT e a concentração diária de $\mathrm{SO}_{2}$ na estação Santa Casa. Em relação ao $\mathrm{O}_{3}$, obteve-se coeficiente de regressão negativo e significativo entre o número de $\mathrm{ObT}$ e a concentração diária de $\mathrm{O}_{3}$ na estação Praça Ouvidor Pardinho, o que parece ser incoerente dada a sua condição de poluente atmosférico.

Destaca-se que, geralmente, os trabalhos relacionando poluição do ar e o número de óbitos foram realizados em cidades com problemas de elevada poluição do ar. Durante o período de estudo, o município de Curitiba atendeu aos padrões primários de qualidade do ar, conforme estabelecidos em CONAMA (1990), especificamente no que se refere ao $\mathrm{SO}_{2}$ e ao PTS, o que implica na questão se existe realmente um valor limite de concentração de um determinado poluente atmosférico abaixo do qual a população, no caso de idosos, estaria segura em relação aos efeitos da poluição do ar sobre a saúde humana.

É importante ressaltar que o presente trabalho se propôs a verificar a existência de relação significativa entre a concentração de poluentes atmosféricos e a mortalidade de idosos. Uma limitação neste tipo de estudo é que não há observação individual, nem a constatação de que algum óbito ocorreu efetivamente devido à elevação da concentração de poluentes na atmosfera.

\section{Referências}

ACKERMANN-LIEBRICH, U.; LEUENBERGER, P.; SCHUWARTZ, J. et al. Lung function and long term exposure to air pollutants in Switzerland. Study on air pollution and lung diseases in adults (SAPALDIA) Team. American Journal of Respiratory and Critical Care Medicine, v. 155, n. 1, p. 122-129, 1997.

ARRUDA, R.J. Associação entre poluição atmosférica e internações hospitalares por doenças respiratórias em crianças, adolescentes e idosos na cidade de Cubatão entre 1997 e 2004. Dissertação (mestrado). Universidade Católica de Santos, 2008.

BAKONYI, S.M.C.; DANNI-OLIVEIRA, I.M.; MARTINS, L.C. et al. Poluição atmosférica e doenças respiratórias em crianças na cidade de Curitiba, PR. Revista Saúde Pública, v. 38 , n. 5, p. 695-700, 2004.

BAKONYI, S.M.C. Fatores socioeconômicos e ambientais e doenças respiratórias em Curitiba/PR. Dissertação (doutorado). Departamento de Geografia da Universidade Federal do Paraná, 2009. 218 p.
BRASIL. Lei Federal $n^{\circ} 10.741$ de $1^{\circ}$ de outubro de 2003. Institui o Estatuto do Idoso e dá outras providências. Publicada no Diário Oficial da União em 03.10.2003.

BRAUN, S. Influências meteorotrópicas nas doenças cardiovasculares na cidade de São Paulo - 1996 a 2000. Dissertação (doutorado). Universidade de São Paulo, 2003

CANÇADO, J.E.D.; BRAGA, A.; PEREIRA, L.A.A. et al. Repercussões clínicas da exposição à poluição atmosférica. Jornal Brasileiro de Pneumologia, v. 32, Suppl. 2, p. S5-S11, 2006.

CLANCY, L.; GOODMAN, P.; SINCLAIR, H. et al. Effect of air-pollution control in deaths rates in Dublin, Ireland: an intervention study. The Lancet. v. 360, n. 9341, p. 1210-1214, 2002.

CONAMA. Resolução 03. Dispõe sobre a qualidade do ar e estabelece os padrões de qualidade do ar. Conselho Nacional do Meio Ambiente, 1990. 
CONCEIÇÃO, G.M.S.; SALDIVA, P.H.N.; SINGER, J.M. Modelos MLG e MAG para análise da associação entre poluição atmosférica e marcadores de morbi-mortalidade: uma introdução baseada em dados da cidade de São Paulo. Revista Brasileira de Epidemiologia, v. 4, n. 3, p. 206-219, 2001.

DANNI-OLIVEIRA, I.M. Poluição do ar como causa de morbidade e mortalidade da população urbana. RA 'EGA: O espaço geográfico em análise, n. 15, p. 113-126, 2008.

DAUMAS, R.P.; MENDONÇA, G.A.S.; LEÓN, A.P. Poluição do ar e mortalidade em idosos no município do Rio de Janeiro: análise de série temporal. Cadernos de Saúde Pública, v. 20, n.1, pp. 311-319, 2004.

DOCKERY, D.W.; POPE, C.A.; XU, X. et al. An association between air pollution and mortality in six U.S. cities. New England Journal of Medicine, v. 329, n. 24, p. 1753-1759, 1993.

FIELD, A. Descobrindo a estatística usando o SPSS. 2a ed. Porto Alegre: Artmed, 2009. 688 p.

FILLEUL, L.; Le TERTRE, A.; BALDI, I. et al. Difference in the relation between daily mortality and air pollution among elderly and all-ages populations in southwestern France. Environmental Research, v. 94, n. 3, p. 249-253, 2004.

FREITAS, C.; BREMNER, A.S.; GOUVEIA, N. et al. Internações e óbitos e sua relação com a poluição atmosférica em São Paulo, 1993 a 1997. Revista Saúde Pública, v. 38, n. 6, p. 751-757, 2004.

HASTIE, T.J.; TIBSHIRANI, R.J. Generalized additive models. London: Chapman and Hall, 1990. 335 p.

JUNGER, W.L.; LEON, A.P.; MENDONÇA, G.A.S. Associação entre a mortalidade diária por câncer de pulmão e poluição do ar no município do Rio de Janeiro: um estudo ecológico de séries temporais. Revista Brasileira de Cancerologia, v. 51, n.2, p. 111-115, 2005.
KATSOUYANNI, K.; ZMIROU, D.; SPIX, C. et al. Short-term effects of air pollution on health: a European approach using epidemiological time-series-data: the APHEA project: background, objectives, design. European Respiratory Journal, v. 8, n. 6, p. 1030-1038, 1995.

KINNEY, P. L.; OZKAYNAK, H. Associations of daily mortality and air pollution in Los Angeles County. Environmental Research Journal. v. 54 n.2, p. 99-120, 1991.

OMS. Classificação estatística de doenças e problemas relacionados à saúde - décima revisão. Tradução do centro colaborador da OMS para a classificação de doenças em português. 8a ed. São Paulo: EDUSP, 2000.

OSTRO, B.; BROADWIN, R.; GREEN, S. et al. Fine particulate air pollution and mortality in nine California counties: results from CALFINE. Environmental Health Perspectives, v. 114, n. 1, p. 29-33, 2006.

SALDIVA, P. H.; POPE, C.A. III; SCHWARTZ, J. et al. Air pollution and mortality in elderly people: a time series study in São Paulo, Brazil. Archives of Environment Health, v. 50, n. 2, p. 159-163, 1995.

SCHWARTZ, J.; MARCUS, A. Mortality and air pollution J London: a time series analysis. American Journal of Epidemiology, v. 131 p. 185-194, 1990.

SICARD, P.; LESNE, O.; ALEXANDRE, N. et al. Air quality trends and potential health effects: development of an aggregate risk index. Atmospheric Environment, v. 45, n. 5, p. 1145-1153, 2011.

TADANO, Y.S.; UGAYA, C.M.L.; FRANCO, A.T. Método de regressão de Poisson: metodologia para avaliação do impacto da poluição atmosférica na saúde populacional. Ambiente \& Sociedade, v. 12, n. 2, p. $241-255,2009$

TRESMONDI, A.C.C.L.; BELI, E.; TOMAZ, E. et al. Concentração de material particulado inalável MP10 em Espírito Santo do Pinhal - SP. Revista Engenharia Ambiental, v. 5, n. 1, p. 133-144, 2008. 